\title{
Effect of flexion degrees in elbow joint on muscle activation of the extensor carpi radialis and biceps brachii muscles in healthy young adults
}

\author{
Gap-Cheol Kim, Sujin Hwang \\ Department of Physical Therapy, Division of Health Science, Baekseok University, Cheonan, Republic of Korea
}

Objective: Chronic lateral epicondylitis is a condition which becomes sore and tender on the lateral side of the elbow joint damaged from overuse and repetitive use of the extensor muscles of the forearm. The purpose of this study was to investigate the effects of flexion degrees in the elbow joint on extensor carpi radialis longus and brevis and biceps brachii muscles in individuals with healthy young adults. The main purpose of this study was to suggest the feasibility of optimal elbow angle during therapeutic eccentric exercise with resistance for strengthening of wrist extensors.

Design: Cross-sectional study.

Methods: Thirty health young adults (male 15, female 15) participated in this study. This study measured muscle activation in four different conditions of elbow flexion, $0^{\circ}, 30^{\circ}, 60^{\circ}$, and $90^{\circ}$ during eccentric exercise with weight loading in wrist extensors, extensor carpi radialis longus and brevis and biceps brachii muscles using surface electromyography.

Results: The muscle activation of extensor carpi radialis showed a negative relationship with the degrees of elbow joint flexion. With increasing elbow flexion angles, the ECRL muscle activation amount was significantly lower $(p<0.05)$. In contrast, the muscle activation of the ECRB muscle activation amount was significanlty higher $(p<0.05)$.

Conclusions: This study suggests that the eccentric exercise of wrist extension with selected activation of wrist extensor muscles according to elbow flexion positions, and suggests that the extensor carpi radialis longus and brevis will need to be strengthened for preventing and treating chronic lateral epicondylitis regardless of degrees of elbow joint flexion.

Key Words: Elbow joint, Muscle contraction, Muscles, Wrist joint

\section{Introduction}

Chronic lateral epicondylitis is a condition, which becomes sore and tender on the lateral side of the elbow joint, and it is known as "tennis elbow" but it is usually not associated with actually playing tennis [1]. Chronic lateral epicondylitis is based on excessive and repetitive use of upper extremity, particularly the extensor muscles of the forearm in daily and functional activities such as reaching, grasping, and manipulation [1]. Therefore, chronic lateral epicondylitis is relatively a common condition that can affect persons with repetitive movement of upper extremity such as table task workers, musicians, as well as homemakers [2].

Chronic lateral epicondylitis involves the proximal tendons of the wrist extensors from a pathomechanical perspective, and extensor carpi radialis is particularly subject to increased stress during functional activities requiring powerful grasping [3]. Clinical rehabilitation settings have supported that therapeutic eccentric exercise is an effective therapeutic approaches to reduce the symptoms associated with overuse syndrome such as chronic lateral epicondylitis $[4,5]$. Previous studies have noted alternative activation, increased or decreased activation of the extensor carpi radialis, but they have not noted the extensor carpi radialis brevis

Received: 16 August, 2016 Revised: 6 September, 2016 Accepted: 7 September, 2016

Corresponding author: Sujin Hwang

Department of Physical Therapy, Division of Health Science, Baekseok University, 76 Munam-ro, Dongnam-gu, Cheonan 31065 , Republic of Korea Tel: 82-41-550-2309 Fax: 82-41-550-2829 E-mail: ptsue@daum.net

(c) This is an Open-Access article distributed under the terms of the Creative Commons Attribution Non-Commercial License (http://creativecommons.org/licens es/by-nc/4.0) which permits unrestricted non-commercial use, distribution, and reproduction in any medium, provided the original work is properly cited.

Copyright $@ 2016$ Korean Academy of Physical Therapy Rehabilitation Science 
(ECRB) although chronic lateral epicondylitis is affected to increase pain at the insertion site of the ECRB muscle, middle finger, and during wrist extension $[4,6,7]$.

One of the important problems in chronic lateral epicondylitis is alternative muscle activation in wrist extensors, and another problem that is not considered but necessary for proper therapeutic exercise approaches to appropriately activate the wrist extensors. This study considered whether the therapeutic eccentric exercise with weight loading on wrist extensors, extensor carpi radialis longus (ECRL) and brevis is affective or not. This study also conducted on the effectiveness of degrees of elbow joint flexion on amount of muscle activation in wrist extensors during the therapeutic eccentric exercise with weight loading. The purpose of this study was to investigate the effects of flexion degrees in elbow joint on extensor carpi radialis and biceps brachii muscles in individuals with healthy young adults. The main purpose of this study was to suggest the feasibility of optimal elbow angle during therapeutic eccentric exercise with resistance for strengthening of wrist extensors.

Table 1. General characteristics of the participants of this study

$(\mathrm{N}=30)$

\begin{tabular}{lrcr}
\hline \multirow{2}{*}{ Variable } & \multicolumn{3}{c}{ Participant } \\
\cline { 2 - 4 } & Male $(\mathrm{n}=15)$ & Female $(\mathrm{n}=15)$ & \multicolumn{1}{c}{ Total } \\
\hline Age $(\mathrm{y})$ & $22.8(2.14)$ & $22.13(0.91)$ & $22.46(1.52)$ \\
Height $(\mathrm{cm})$ & $171.9(7.57)$ & $164.3(9.67)$ & $168.01(8.64)$ \\
Weight $(\mathrm{kg})$ & $72.5(6.61)$ & $51.65(6.15)$ & $62.07(6.38)$ \\
\hline
\end{tabular}

Values are presented as mean (SD).

\section{Methods}

\section{Subjects}

Thirty healthy young adults (male 15 , female 15 ) participated in this study. Participants conducted the purpose and method of this study before participating and written consent to research participation were given spontaneously. The inclusion criteria were as follows: persons without any pain in upper extremity and upper body before last six months, persons without taking any medication and any plastic surgery at upper and low extremities, and persons without any neurological lesion. Participants who had a skin problem affected by attaching the surface electrode were excluded in this study. The characteristics of participants are shown in Table 1 .

\section{Procedure}

This study measured muscle activation in four different conditions of elbow flexion, $0^{\circ}, 30^{\circ}, 60^{\circ}$, and $90^{\circ}$ during eccentric exercise with weight loading (Figure 1). First of all, the participants were instructed to sit in a standard sitting position in a chair for eccentric exercises in front of a desk with the shoulder joint in $90^{\circ}$ flexion, and forearm supinated and were asked to relax as much as possible. The participants performed wrist joint extension $60^{\circ}$ while holding a $2 \mathrm{~kg}$ dumbbell for 10 seconds, repeating 3 times, while maintaining each condition of elbow flexion. Previously mentioned, after eccentric exercises of wrist extension at $60^{\circ}$, the participants were measured of their muscle activations of the ECRL, ECRB, and biceps brachii while holding the wrist in eccentric contraction at $60^{\circ}$ wrist extension for 10 seconds.

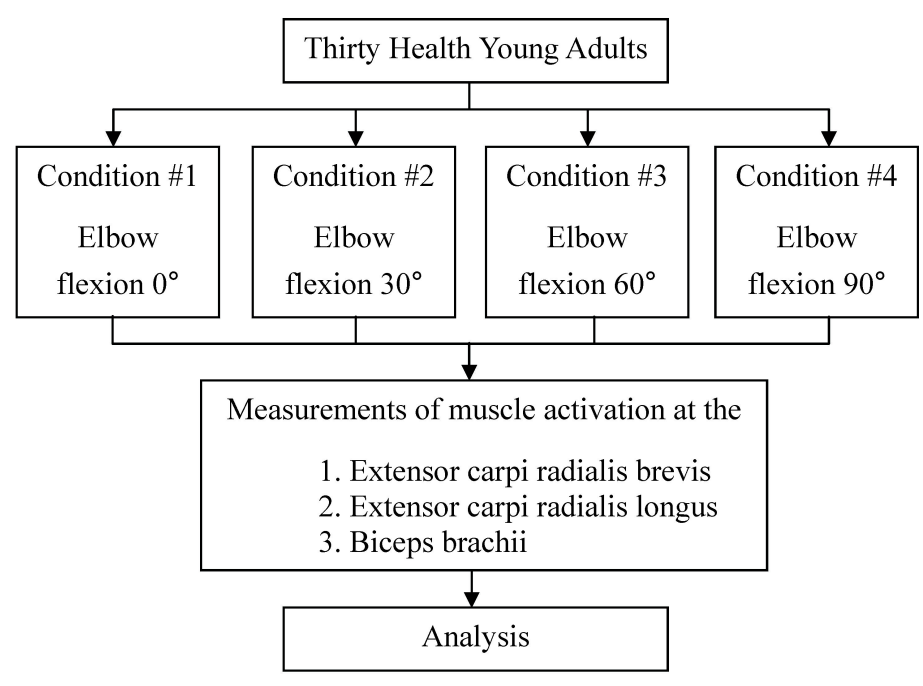

Figure 1. The experimental procedure of this study. 


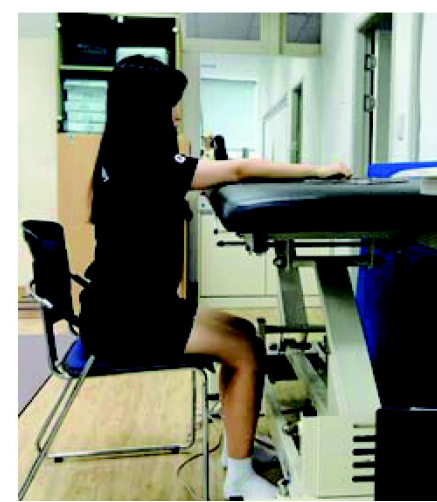

Side view

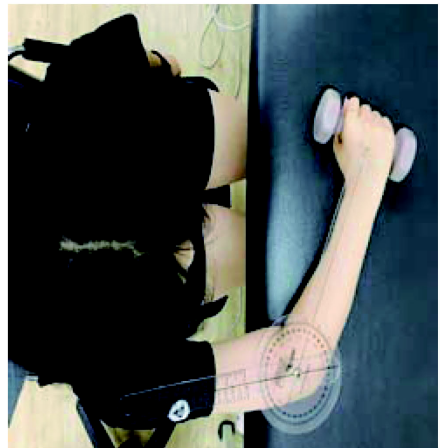

Condition \#3, $60^{\circ}$

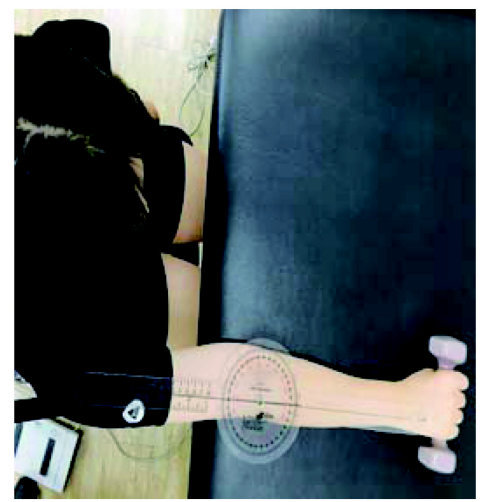

Condition $\# 1,0^{\circ}$

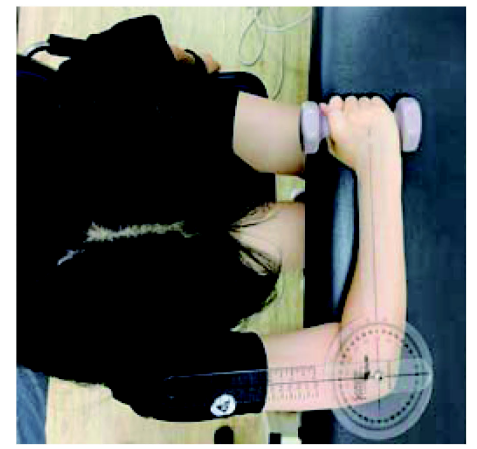

Condition $\# 4,90^{\circ}$

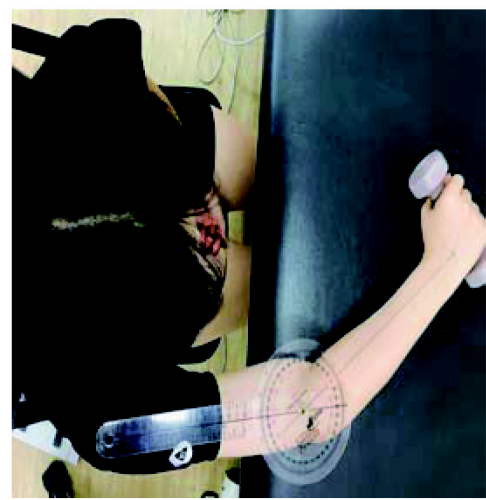

Condition \#2, $30^{\circ}$

Figure 2. Side view and four different conditions of elbow flexion degrees.

Muscle activation was measured at 5 seconds, at the middle of 10 seconds, 3 times, and there was a five minute interval between each muscle contraction to avoid muscle fatigue. The filtered muscle activation was normalized by dividing the observed electromyography value by the maximum voluntary isometric contraction. Mean values of maximal voluntary isometric contraction were normalized and was used for analysis in this study (Figure 2).

\section{Experimental equipment}

This study used surface electromyography device (Trigno; Delsys Inc., Boston, MA, USA) to measure muscle activation of wrist extensors and biceps brachii according to four different conditions of elbow flexion positions. The signal sampling rate of the electromyography was set to $2,000 \mathrm{~Hz}$, and the electromyography data was analyzed by Trigno Base 4.0 software. On the wrist extensors, electrode placement was on the muscle mass approximately $5 \mathrm{~cm}$ distal from the lateral epicondyle of the humerus. On the ECRL, 2 $\mathrm{cm}$ apart from the electrode of the ECRL over the muscle mass that emerge with the electrodes running in the direction of the muscle fibers at ECRB. Biceps brachii was measured over the muscle belly, approximately two-thirds of the distance between the shoulder and the elbow on the anterior surface of the humerus in the vertical plane [8].

\section{Statistical analysis}

The dependent variables were muscle activation of ECRL, ECRB, and biceps brachii, and the independent variable was degrees of elbow joint flexion in this study. Descriptive statistics was used to define the common characteristics (mean, standard deviation, and range) of the participants of this study. Repeated measures ANOVA used to analyze the muscle activation of biceps brachii and ECRL and brevis according to four different conditions in the elbow joint. This study also used Fisher's least significant difference as the post-hoc analysis. All statistical calculations were performed using the IBM Statistical Package for the Social Sciences 20.0 statistical package (IBM Co., Armonk, NY, USA). The significance level was set at $\alpha=0.05$. 
Table 2. Wrist extensors and biceps brachii muscle activation in four different condition with elbow flexion

\begin{tabular}{lrrrrrr}
\hline \multirow{2}{*}{ Variable } & \multicolumn{9}{c}{ Elbow flexion degree $\left({ }^{\circ}\right)$} & \multirow{2}{*}{ F } & $p$ & \\
\cline { 2 - 4 } & \multicolumn{1}{c}{0} & \multicolumn{1}{c}{30} & 60 & 90 & & \\
\hline Biceps brachii (\%MVIC) & $8.9(0.2)$ & $9.1(5.2)$ & $9.1(4.8)$ & $9.3(4.6)$ & 1.40 & 0.264 \\
ECRL (\%MVIC) & $16.7(8.3)$ & $14.9(6.7)$ & $14.6(7.3)$ & $14.1(7.2)$ & 20.111 & $<0.001$ \\
ECRB (\%MVIC) & $14.3(6.9)$ & $15.3(7.1)$ & $15.9(7.2)$ & $16.8(7.9)$ & 17.682 & $<0.001$ \\
\hline
\end{tabular}

Values are presented as mean (SD).

MVIC: maximal voluntary isometric contraction, ECRL: extensor carpi radialis longus, ECRB: extensor carpi radialis brevis.

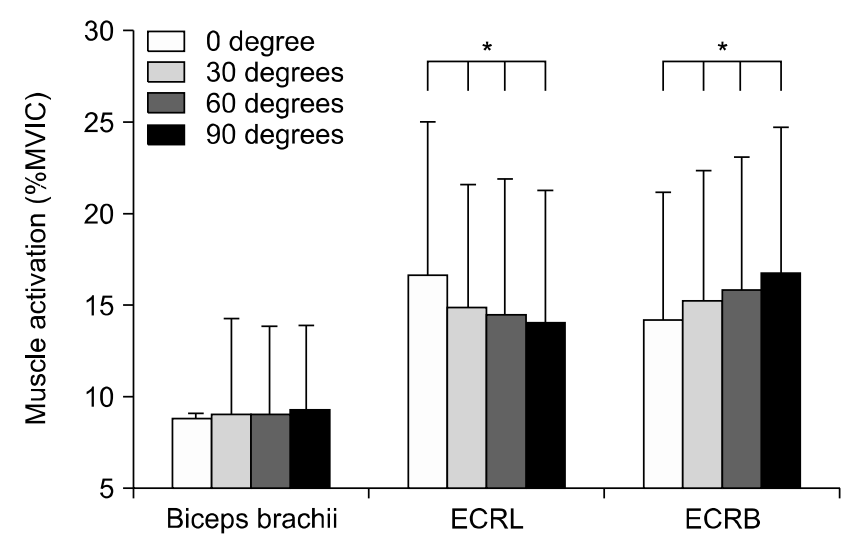

Figure 3. \% Maximal voluntary isometric contraction (MVIC) of biceps brachii, extensor carpi radialis longus (ECRL), and extensor carpi radialis brevis (ECRB) in four different condition with elbow joint flexion. *Significantly difference between each degree conditions $(p<0.05)$.

\section{Results}

The muscle activation of biceps brachii was not significantly different in all four different conditions of elbow flexion. The ECRL showed a decrease in muscle activation, as the degrees of elbow joint flexion increased. The more flexed posture the elbow joint was, muscle activation the ECRB increased (Table 2, Figure 3).

\section{Discussion}

This study evaluated the effect of elbow flexion on wrist extensors and biceps brachii muscle in healthy young adults. The results of this study show that the ECRL decreased according to increasing flexion of the elbow joint, but ECRB was increased with an increase in elbow flexion. Therefore, muscle activation of the ECRB increase to reinforce the wrist extension in inverted proportion to muscle activation of the ECRL during eccentric contraction regardless of the degrees of elbow joint flexion.
Previous studies have reported the effectiveness of therapeutic approaches such as medication, electrical and thermal modalities, cold massage, cross friction massage, and steroid transcutaneous injection on chronic lateral epicondylitis [4,5,9-11]. However, these therapeutic approaches would have temporary effects and has some side effects. Therefore, it is necessary to strengthen wrist extensor muscles themselves. Chronic lateral epicondylitis occurs from repetitive forearm pronation and supination with wrist hyperextension so the age of this common occurrence in persons is not selective and it is the most common disorder in musculoskeleral conditions [12]. A stretching technique of wrist extensors for lateral epicondylitis is generally pulling the wrist into flexion with the elbow in extension and forearm in pronation [13]. Shirato et al. [14] reported the effectiveness of the stretching technique has not been clarified in the ECRB and extensor digitorum communis (EDC). They have emphasized the therapeutic exercise of ECRB for lateral epicondylitis. Nimura et al. [12] evaluated to identify the unique anatomical characteristics of the ECRB origin and points of differentiation from other wrist extensors. They reported the anterior part of the ECRB origin was delicate, because the ECRB origin was purely tendinous and the attachment of the articular capsule was thin compared with that of the posterodistal attachment. The thin attachment could be an initial factor leading to the development of lateral epicondylitis. According to the previous studies, the ECRB is the most important factor causing lateral epicondylitis, and then ECRB would be taken the proper management to prevent and treat the chronic lateral epicondylitis in clinical rehabilitation settings [7,12]. However, the established eccentric exercise for chronic lateral epicondylitis has not considered the ECRB except ECRL and EDC muscles [1,4]. The results of this study also show the important consideration of ECRB in wrist extension exercise with weight loading. Depending on the flexion angle of the elbow joint, the fulcrum point and angle and distance from the fulcrum point to 
muscle fibers are different in mechanical kinesiology's view, so that the muscle activation of the ECRB was different depending on the increase of flexion degrees of the elbow joint, and the ECRB muscle activation increased to decrease the ECRL activation. Therefore, the ECRB muscle activation would be to reinforce the ECRL muscle activation according to increase elbow joint flexion during wrist extension. There is important muscle activation because most table tasks for functional activities take several positions of elbow flexion from $0^{\circ}$ to $90^{\circ}$. The strengthening of the ECRL and ECRB for preventing and treating chronic lateral epicondylitis regardless of degrees of elbow joint flexion is important. This study suggests that eccentric exercises of wrist extension was activated of wrist extensor muscles according to elbow flexion positions.

This study has been showed several limitations. The therapeutic effect of strengthening the ECRB and ECRL muscles will be used in future study. The other wrist extensors such as EDC will need to be investigated in the preventive and therapeutic effects for lateral epicondylitis likewise ECRB and ECRL muscles. This study involved only healthy young adults and future studies will need to involve the persons with chronic lateral epicondylitis to find evidence of preventing and treating the epicondylitis throughout eccentric exercises of the ECRB and ECRL muscles.

\section{Conflict of Interest}

The authors declared no potential conflicts of interest with respect to the authorship and/or publication of this article.

\section{References}

1. Croisier JL, Foidart-Dessalle M, Tinant F, Crielaard JM, Forthomme B. An isokinetic eccentric programme for the management of chronic lateral epicondylar tendinopathy. Br J Sports Med 2007; 41:269-75
2. Page P. A new exercise for tennis elbow that works! N Am J Sports Phys Ther 2010;5:189-93.

3. Ellenbecker TS, Roetert EP, Riewald S. Isokinetic profile of wrist and forearm strength in elite female junior tennis players. Br J Sports Med 2006;40:411-4.

4. Wen DY, Schultz BJ, Schaal B, Graham ST, Kim BS. Eccentric strengthening for chronic lateral epicondylosis: a prospective randomized study. Sports Health 2011;3:500-3.

5. Cullinane FL, Boocock MG, Trevelyan FC. Is eccentric exercise an effective treatment for lateral epicondylitis? A systematic review. Clin Rehabil 2014;28:3-19.

6. Tyler TF, Thomas GC, Nicholas SJ, McHugh MP. Addition of isolated wrist extensor eccentric exercise to standard treatment for chronic lateral epicondylosis: a prospective randomized trial. J Shoulder Elbow Surg 2010;19:917-22.

7. Drake ML, Ring DC. Enthesopathy of the extensor carpi radialis brevis origin: effective communication strategies. J Am Acad Orthop Surg 2016;24:365-9.

8. Criswell E, Cram JR. Cram's introduction to surface electromyography. 2nd ed. Sudbury (MA): Jones and Bartlett; 2011.

9. Sims SE, Miller K, Elfar JC, Hammert WC. Non-surgical treatment of lateral epicondylitis: a systematic review of randomized controlled trials. Hand (N Y) 2014;9:419-46.

10. Dingemanse R, Randsdorp M, Koes BW, Huisstede BM. Evidence for the effectiveness of electrophysical modalities for treatment of medial and lateral epicondylitis: a systematic review. Br J Sports Med 2014;48:957-65.

11. Smidt N, Assendelft WJ, van der Windt DA, Hay EM, Buchbinder R, Bouter LM. Corticosteroid injections for lateral epicondylitis: a systematic review. Pain 2002;96:23-40.

12. Nimura A, Fujishiro H, Wakabayashi Y, Imatani J, Sugaya H, Akita K. Joint capsule attachment to the extensor carpi radialis brevis origin: an anatomical study with possible implications regarding the etiology of lateral epicondylitis. J Hand Surg Am 2014;39:219-25

13. Martinez-Silvestrini JA, Newcomer KL, Gay RE, Schaefer MP, Kortebein P, Arendt KW. Chronic lateral epicondylitis: comparative effectiveness of a home exercise program including stretching alone versus stretching supplemented with eccentric or concentric strengthening. J Hand Ther 2005;18:411-9, quiz 420.

14. Shirato R, Wada T, Aoki M, Iba K, Kanaya K, Fujimiya M, et al. Effect of simultaneous stretching of the wrist and finger extensors for lateral epicondylitis: a gross anatomical study of the tendinous origins of the extensor carpi radialis brevis and extensor digitorum communis. J Orthop Sci 2015;20:1005-11. 